\title{
Mach Number Effect on Supersonic Drag Reduction using Repetitive Laser Energy Depositions over a Blunt Body*
}

\author{
Akira Iwakawa, ${ }^{1) \dagger}$ Tatsuro Shoda, ${ }^{1)}$ Ryosuke MaJIMA, ${ }^{1,2)}$ Son Hoang Pham, ${ }^{1)}$ and Akihiro SASOH ${ }^{1)}$ \\ ${ }^{1)}$ Department of Aerospace Engineering, Nagoya University, Nagoya, Aichi 464-8603, Japan \\ ${ }^{2)}$ Currently, Aero-Engine, Space and Defense Business Area, IHI Corporation, Nishitama, Tokyo 190-1297, Japan
}

\begin{abstract}
Supersonic drag reduction performance using repetitive pulse energy depositions over blunt bodies was experimentally studied under two Mach numbers. The normalized drag reduction and energy deposition efficiency of Mach-1.92 over a 10-mm-dia. blunt-cylinder model were $8 \%$ and 1.2 at most, respectively. On the other hand, these values at Mach-3.20 over the same model were $22 \%$ and 6.2 , respectively. The shock-wave deformation period using single-pulse energy deposition at Mach-3.20 was $64 \mu \mathrm{s}$. This duration was shorter than that of $80 \mu \mathrm{s}$ at Mach-1.92, but the deformation magnitude on the model center axis of $40 \%$ at Mach-3.20 was larger than that of 15\% at Mach-1.92. These experimental characteristics were consistent as solutions of the Riemann problem. Moreover, a drag reduction performance was much improved with a larger model diameter of $20 \mathrm{~mm}$. Therefore, it has been experimentally demonstrated that the drag reduction performance due to energy deposition improves much at a high Mach number and with large model dimensions.
\end{abstract}

Key Words: Aerodynamics, Shock-waves, Wind Tunnel Testing, Drag Reduction, Energy Deposition

\section{Nomenclature}

A: model cross-sectional area against flow

$A_{\mathrm{b}}$ : cross-sectional area of thermal bubble

$c_{\mathrm{p}}$ : specific heat at constant pressure

$d$ : model diameter

$D$ : drag

$D_{0}$ : baseline drag without energy deposition

$E$ : pulse energy of energy deposition

$f_{\mathrm{e}}$ : repetition frequency of energy deposition

$L$ : scale length of energy deposition

$M$ : Mach number

$p$ : pressure

$P_{\mathrm{b}}$ : input power on focal spot

$q_{\mathrm{b}}$ : specific energy input

St: Strouhal number

$t$ : time

$T$ : temperature

$\boldsymbol{u}$ : flow velocity vector

$U$ : upstream flow speed

$\gamma$ : specific heat ratio

$\delta$ : shock stand-off distance

$\Delta C_{\mathrm{D}}$ : normalized drag reduction

$\triangle D$ : drag reduction

$\Delta E$ : pulse energy absorbed by gas

$\varepsilon$ : dimensionless energy of energy deposition

$\eta$ : efficiency of energy deposition

$v$ : kinematic viscosity

$\rho$ : density

$\sigma$ : standard deviation

$\omega$ : vorticity

(C) 2017 The Japan Society for Aeronautical and Space Sciences *Received 6 June 2016; final revision received 10 April 2017; accepted for publication 10 May 2017.

†Corresponding author, iwakawa@nuae.nagoya-u.ac.jp
Subscript
1: upstream flow
2: behind bow shock without energy deposition
3: in thermal bubble
$\mathrm{h}$ : thermal bubble
m: model

\section{Introduction}

Improving aerodynamic performance during supersonic flight by reducing wave drag is still an important issue for re-realizing commercial supersonic transport aircraft. Many shock-wave mitigation approaches such as mechanical methods or non-mechanical methods are suggested. The reduction methods for drag due to the shock-wave have been summarized in detail by Bushnell. ${ }^{1)}$ The energy deposition approach $^{2)}$ is one of the non-mechanical drag reduction approaches, the idea of which was pioneered by Georgievskii and Levin. ${ }^{3)} \mathrm{A}$ large number of the experimental ${ }^{4-13)}$ and numerical studies ${ }^{14-21)}$ about drag reduction using energy deposition can be found. Some of these studies have reported that the energy deposition method is also effective to mitigate the sonic boom applying the shock-wave mitigation effect of energy deposition. ${ }^{22-24)}$

The drag reduction mechanisms enabled by energy deposition have been discussed using computational fluid dynamics. ${ }^{12,15,25)}$ Applying energy deposition a high-temperature low-density bubble, a so-called "thermal bubble" generated upstream, and the shock-wave is weakened when interacting with the bubble; the acoustic impedance of which is lower than that of the post-shock flow. As a result, the shock-wave extrudes upstream (lens effect ${ }^{26)}$ ). This interaction is followed by the generation of a vortex ring due to the baroclinic effect. ${ }^{15,27)}$ The pressure in the shock layer is mitigated in the vortex ring, thereby reducing the drag. ${ }^{25)}$ This is the basic 
mechanism of drag reduction using energy deposition.

Large drag reduction can be obtained either by enhancing the vorticity or increasing the residence time of the vortex in the shock layer. The vortex generated by the baroclinic term is obtained as the vector product of a density gradient and a pressure gradient in the vorticity equation:

$$
\frac{d \boldsymbol{\omega}}{d t}=(\boldsymbol{\omega} \cdot \nabla) \boldsymbol{u}-(\nabla \cdot \boldsymbol{u}) \boldsymbol{\omega}+\frac{1}{\rho^{2}} \nabla \rho \times \nabla p+v \nabla^{2} \boldsymbol{\omega}
$$

Strong vorticity is obtained by increasing the density difference across the bubble interface and/or increasing the pressure jump across the shock-wave.

The performance of drag reduction using energy deposition is evaluated by two criteria. One is the magnitude of the drag reduction $\Delta D$ as shown in Eq. (2),

$$
\Delta D=D-D_{0}
$$

where $D$ and $D_{0}$ are drags with and without energy deposition, respectively. Normalized drag reduction can be defined by Eq. (3),

$$
\Delta C_{\mathrm{D}}=\frac{-\Delta D}{\frac{1}{2} \rho U^{2} A}
$$

where $U$ is the flow speed and $A$ is the cross-sectional area of the body.

Another evaluation is done with the efficiency of energy deposition, $\eta{ }^{2}{ }^{2}$ This is expressed using the drag reduction and an input power $P_{\mathrm{b}}$ such that

$$
\eta=\frac{-\Delta D \times U}{P_{\mathrm{b}}}=\frac{\Delta C_{\mathrm{D}} \times \frac{1}{2} \rho U^{3} A}{P_{\mathrm{b}}}
$$

In the case of pulsed energy deposition, $P_{\mathrm{b}}$ can be written using the energy incident on the focal spot in the gas in each pulse $\Delta E$ and the repetition frequency $f_{\mathrm{e}}$, as follows.

$$
P_{\mathrm{b}}=\Delta E f_{\mathrm{e}}
$$

Here, $\Delta E=\varepsilon p L^{3}$, where $\varepsilon$ is a dimensionless energy of the energy deposition per pulse and $L$ is the scale length of energy deposition. Hence, using these equations and $S t=U / f_{\mathrm{e}} L, \eta$ can be written as follows.

$$
\eta=\frac{\gamma}{2} \frac{M^{2}}{S t} \frac{A}{L^{2}} \frac{\Delta C_{\mathrm{D}}}{\varepsilon}
$$

In Eq. (6), of course, the dependence of $\Delta C_{\mathrm{D}}$ on control parameters is the key to obtaining a large value for $\eta$. However, the dependence is not readily obtained through a simple model. This is why experimental data is important. Additionally, $M$ and $S t$ are important control parameters. The dependence of dimensionless pulse repetition frequency, which corresponds to $S t$, for drag reduction has been experimented by Tret'yakov et al. ${ }^{28)}$ From their results, the maximum drag reduction is achieved at $S t \approx 1$. From the dependences of Mach number and model dimensions in Eqs. (4) and (6), energy deposition performance is expected to be improved at an even higher Mach number and larger model. Although the Mach number effect was investigated by numerical simulation, ${ }^{15)}$ a direct experimental comparison has not been done. The objective of this study is to experimentally investigate the energy deposition drag reduction performance at different Mach numbers.

\section{Effect of Thermal Bubble as a Solution for the Rie- mann Problem}

In a past analysis of supersonic drag reduction using energy deposition, ${ }^{29)}$ the importance of the heat input ratio has already been shown. Here, the Mach number dependence on the pressure field and the induced flow of this phenomena are analyzed based on compressible fluid dynamics. The interaction between a thermal bubble and a bow shock-wave is modeled as a Riemann problem. ${ }^{30)}$ The schematic illustration of the model is shown in Fig. 1. On the center axis of the model, the states of the upstream flow behind the bow shock-wave and in the thermal bubble are labeled as state$1,-2$ and -3 , respectively. A thermal bubble is produced by isochorically depositing an energy of $q$ per unit mass in state-1. Then, the heated region is assumed to isentropically expand to state- 3 so that the pressure in the bubble becomes equilibrated to the surroundings in state- 1 . The following equations are established under the calorically perfect gas.

$$
\begin{gathered}
p_{3}=p_{1} \\
\frac{1}{\gamma-1} R T_{1^{\prime}}=\frac{1}{\gamma-1} R T_{1}+q \\
\frac{p_{1^{\prime}}}{T_{1^{\prime}}}=\frac{p_{1}}{T_{1}} \\
\frac{p_{3}}{p_{1^{\prime}}}=\frac{p_{1}}{p_{1^{\prime}}}=\left(\frac{\rho_{3}}{\rho_{1^{\prime}}}\right)^{\gamma}=\left(\frac{\rho_{3}}{\rho_{1}}\right)^{\gamma} \\
\frac{a_{3}}{a_{1^{\prime}}}=\left(\frac{T_{3}}{T_{1^{\prime}}}\right)^{\frac{1}{2}}=\left(\frac{\rho_{3}}{\rho_{1}}\right)^{\frac{\gamma-1}{2}} \\
\frac{\rho_{3}}{\rho_{1}}=(1+Q)^{-\frac{1}{\gamma}} \\
\frac{a_{3}}{a_{1}}=(1+Q)^{\frac{1}{2 \gamma}}
\end{gathered}
$$

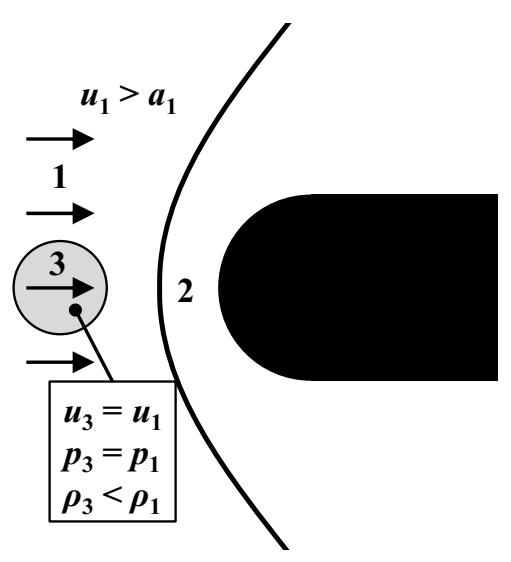

Fig. 1. Schematic illustration of the analysis model. 


$$
\begin{aligned}
\frac{\rho_{3} a_{3}}{\rho_{1} a_{1}} & =(1+Q)^{-\frac{1}{2 \gamma}}<1 \\
Q & =\frac{q}{R T_{1} /(\gamma-1)}
\end{aligned}
$$

From Eq. (14), the acoustic impedance is decreased through energy deposition. Here, for simplification, only the center axis of the model (equal to the axis on the stagnation point) is considered. The following equations are established using the normal shock relation between state- 2 and state- 3 ,

$$
\begin{gathered}
\frac{p_{2}}{p_{3}}=\frac{p_{2}}{p_{1}}=1+\frac{2 \gamma}{\gamma+1}\left(M_{1}^{2}-1\right) \\
\frac{u_{3}}{a_{1}}=M_{1} \\
\frac{u_{2}}{a_{1}}=M_{1}-\frac{2}{\gamma+1}\left(M_{1}-\frac{1}{M_{1}}\right) \\
\frac{\rho_{2}}{\rho_{1}}=\frac{(\gamma+1) M_{1}^{2}}{(\gamma-1) M_{1}^{2}+2} \\
\frac{a_{2}}{a_{1}}=\frac{\left(2 \gamma M_{1}^{2}-\gamma+1\right)^{\frac{1}{2}}\left\{(\gamma-1) M_{1}^{2}+2\right\}^{\frac{1}{2}}}{(\gamma+1) M_{1}}
\end{gathered}
$$

From the normal shock relation,

$$
\begin{gathered}
\frac{\rho_{2} a_{2}}{\rho_{1} a_{1}}>1 \\
\frac{\rho_{3} a_{3}}{\rho_{2} a_{2}}=\frac{\rho_{3} a_{3}}{\rho_{1} a_{1}} \frac{\rho_{1} a_{1}}{\rho_{2} a_{2}}<1
\end{gathered}
$$

From these relations, flow conditions after the interaction $\left(u_{*}, p_{*}\right)$ can be obtained by equating the following equations.

$$
\begin{gathered}
\frac{u_{*}}{a_{1}}-\frac{u_{3}}{a_{1}}=-\frac{a_{3}}{a_{1}}\left(\frac{p_{*}}{p_{1}}-1\right)\left\{\frac{\frac{2}{\gamma(\gamma+1)}}{\frac{p_{*}}{p_{1}}+\frac{\gamma-1}{\gamma+1}}\right\}^{\frac{1}{2}} \\
\frac{u_{*}}{a_{1}}-\frac{u_{2}}{a_{1}}=\frac{2}{\gamma-1} \frac{a_{2}}{a_{1}}\left\{\left(\frac{p_{*}}{p_{2}}\right)^{\frac{\gamma-1}{2 \gamma}}-1\right\}
\end{gathered}
$$

Therefore, $p_{*} / p_{1}$ is obtained applying the function of $Q$ and $M_{1}$.

$$
\frac{p_{*}}{p_{1}}=f\left(Q, M_{1}\right)
$$

where $f$ is obtained as the solution to the implicit equation, Eq. (26). In this way, $p_{*} / p_{2}$ and $u_{*} / a_{1}$ are also obtained as a function of $Q$ and $M_{1}$.

The pressure mitigation effect and induction of counter flow are major effects of energy deposition. The Mach number dependence of the post-shock pressure and that of the normalized flow velocity induced are shown in Figs. 2 and 3 , respectively. The higher the Mach number, the stronger these effects become. Particularly, at a relatively low Mach number lower than 3, the pressure mitigation effect is significant, whereas with a large value of $Q$, the counter-flow induction becomes significant in the higher Mach number region. These results enhance the motivation of examining drag reduction performance with energy deposition at different Mach numbers.

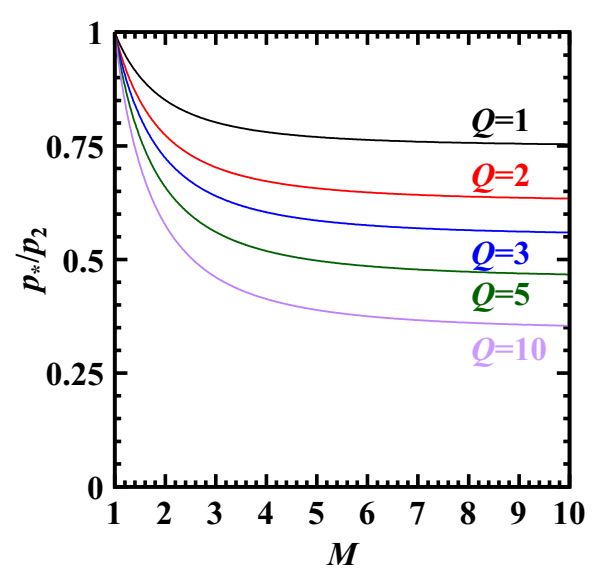

Fig. 2. Mach number dependence of $p_{*} / p_{2}$.

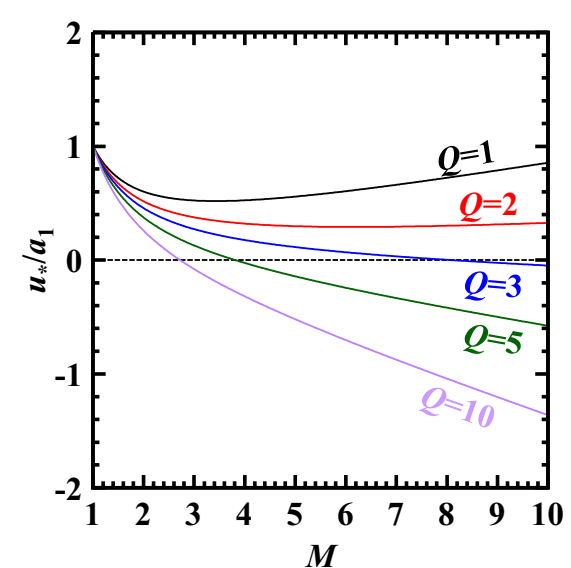

Fig. 3. Mach number dependence of $u_{*} / a_{1}$. 


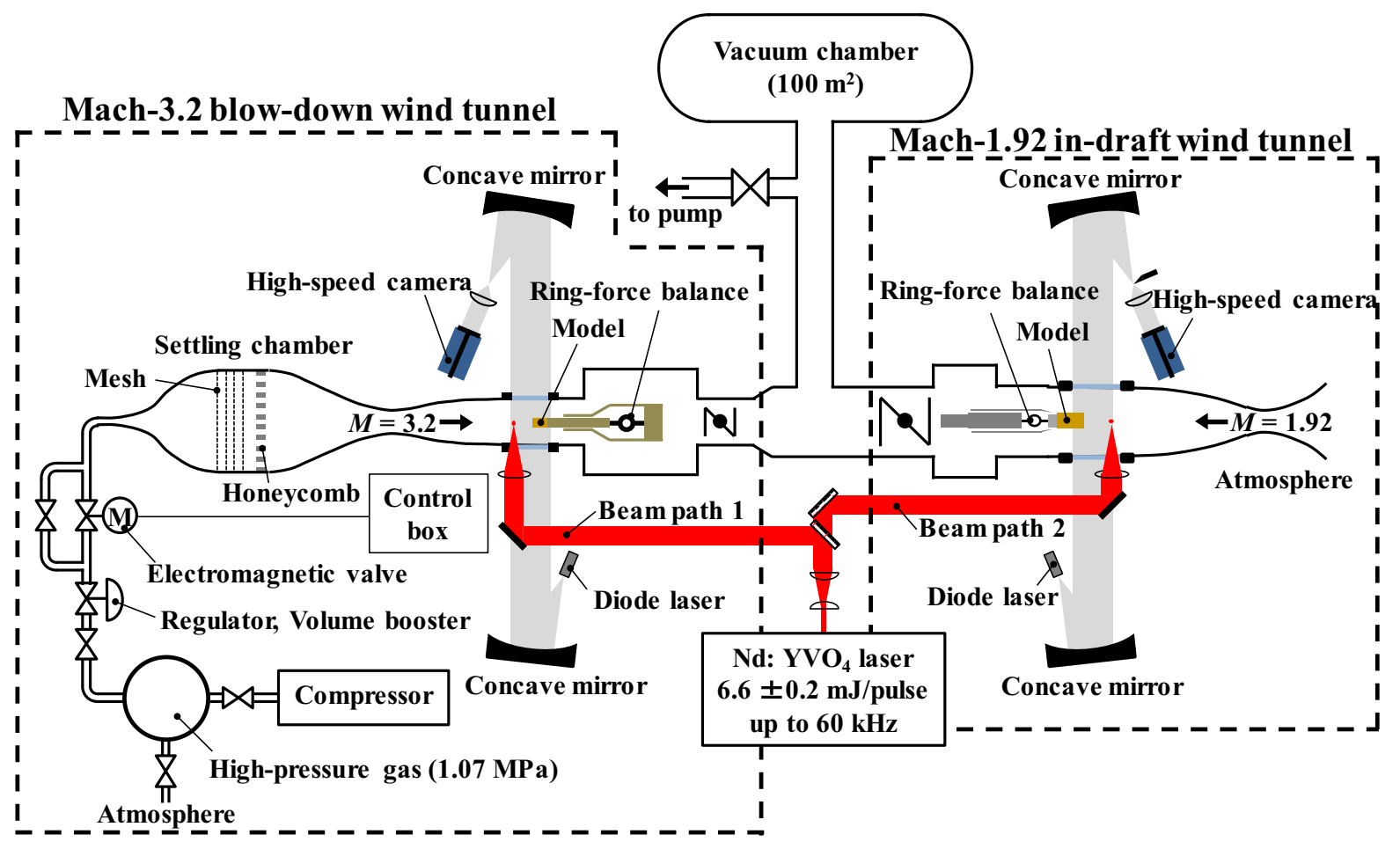

Fig. 4. Schematic view of the experimental apparatus.

One of the beam paths, 1 or 2, is used during an experiment: path 1 is for Mach-3.20, and path 2 is for Mach-1.92.

\section{Experimental Apparatus}

\subsection{Wind tunnel}

Experiments were conducted in an in-draft wind tunnel with a Mach number of 1.92 (Mach-1.92-WT) and a blowdown wind tunnel with a Mach number of 3.20 (Mach3.20-WT). The wind tunnel system is shown in Fig. 4, and specifications of the wind tunnels are shown in Table 1. Both of the wind tunnels were connected to a common dump tank with a volume of $100 \mathrm{~m}^{3}$, which was evacuated down to $10 \mathrm{kPa}$ or lower prior to experiments using a rotary pump with a volume flow rate of $7,500 \mathrm{~L} / \mathrm{min}$.

Mach-1.92-WT is an in-draft wind tunnel with a square test section of $80 \mathrm{~mm} \times 80 \mathrm{~mm}$, and has been used in past studies. $.6,13)$ This wind tunnel is started when the butterfly valve, which was installed downstream behind the test section, is opened. The Mach number, static pressure and static temperature during the experiment were $1.92,13.8 \mathrm{kPa}$ and $162 \mathrm{~K}$, respectively. The test time was extended by increasing the volume of the dump tank in comparison with the time of $5 \mathrm{~s}$ in past studies, and the maximum available test time is $20 \mathrm{~s}$. In these experiments, the test time was set to $3 \mathrm{~s}$.

Mach-3.20-WT was newly installed to conduct the experiment with repetitive laser energy deposition. The supersonic wind tunnel installed in JAXA was referenced for the tunnel design. It consists of a high-pressure reservoir of up to 1.1 MPa, a pressure regulator, an electromagnetic valve, a settling chamber, a Laval nozzle, a test section, a butterfly valve and the dump tank. Four sheets of metal mesh and a honeycomb were installed in the settling chamber. The upper and lower portions of the nozzle were corrected against
Table 1. Specifications of wind tunnels.

\begin{tabular}{lccc}
\hline \multicolumn{1}{c}{ Name } & Unit & Mach-1.92-WT & Mach-3.20-WT \\
\hline Mach number, $M$ & & 1.92 & 3.20 \\
Type & & In-draft & Blow-down \\
Test time & $\mathrm{s}$ & 5 & 3 \\
Static pressure, $p_{1}$ & $\mathrm{kPa}$ & 13.8 & 12.5 \\
Static temperature, $T_{1}$ & $\mathrm{~K}$ & 163 & 107 \\
Density & $\mathrm{kg} / \mathrm{m}^{3}$ & 0.29 & 0.41 \\
Flow speed, $U$ & $\mathrm{~m} / \mathrm{s}$ & 495 & 661 \\
Acoustic speed, $a_{1}$ & $\mathrm{~m} / \mathrm{s}$ & 258 & 207 \\
Cross-sectional area & $\mathrm{mm}$ & $6400(80 \times 80)$ & $1600(40 \times 40)$ \\
$\quad$ at test section & & & \\
\hline
\end{tabular}

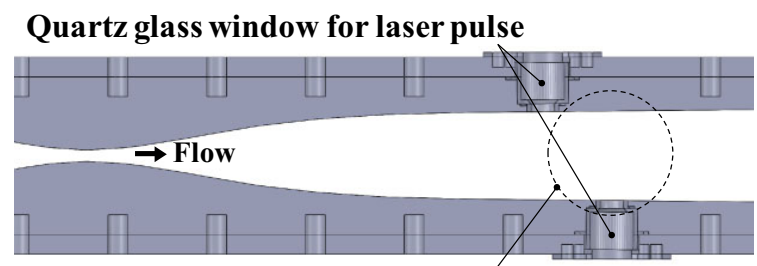

BK7 window for visualization

Fig. 5. Test section of Mach-3.2-WT.

boundary layer development. As shown in Fig. 5, quartz glass windows were installed in the vertical direction upstream of the test section to send repetitive pulsed laser beams. The inner surfaces of these windows were finished flush with the nozzle surface so as not to disturb the test flow. The cross-sectional area of the test section of this wind tunnel was $40 \mathrm{~mm} \times 40 \mathrm{~mm}$ and the test time, which was determined by the volume of the high-pressure reservoir, was $3 \mathrm{~s}$. Prior to the experiments, the Mach number of the test 
flow was calibrated using a Rayleigh Pitot tube formula and Pitot pressure measurement using a Pitot rake. At the test section, the flow Mach number was $3.2 \pm 0.2$ in a central rectangle with a width of $20 \mathrm{~mm}$ and a height of $30 \mathrm{~mm}$. The static pressure and static temperature were $12.7 \mathrm{kPa}$ and $107 \mathrm{~K}$, respectively. While the static pressure was measured on the wall, the static temperature was evaluated using the temperature measured by a thermocouple installed in the settling chamber and assumed to be the stagnation temperature. Before the experiments, the test section was depressurized and the test flow was supplied from the reservoir when the valve at the upstream location of the test section was opened.

\subsection{Measurement}

The flow fields during the experiment were visualized using the Schlieren method with a high-speed camera (Shimadzu Corporation, HPV-1, $312 \times 260$ pixels, 1 Mfps maximum, 102 frames). A synchronized pulse diode laser (Cavitar Ltd., CAVILUX Smart, wavelength: $640 \mathrm{~nm}$, pulse duration: $10 \mathrm{~ns}$ minimum) was introduced as the light source. The pulse duration of the light source was set to $10 \mathrm{~ns}$ in all experiments; both the time resolution and brightness of the images were much improved.

The force exerted to the model was measured by a ringforce balance system. ${ }^{31)}$ In this paper, "drag" refers to a force acting only on the frontal surface of the model.

\subsection{Experimental conditions}

The energy was supplied by a highly repetitive pulse laser (EdgeWave GmbH Innovative Laser Solutions, HD40I-E $\mathrm{Nd}: \mathrm{YVO}_{4}$ laser, wavelength: $1,064 \mathrm{~nm}$, repetition frequency: up to $100 \mathrm{kHz}$, average power: up to $400 \mathrm{~W}$ ). A $6 \mathrm{~mm} \times$ $6 \mathrm{~mm}$ beam from the laser was expanded to $15 \mathrm{~mm} \times$ $15 \mathrm{~mm}$ using a beam expander. The expanded beam was then reflected by three (Mach-1.92-WT) or four (Mach3.20-WT) dielectric-coated mirrors and delivered to the wind tunnel. The thermal bubble was generated upstream of the flow by focusing the laser beam using a LightPath $^{\circledR}$ GRADIUM $^{\circledR}$ lens with a focal length of $60 \mathrm{~mm}$. The repetition frequency of the laser pulse was set to $0-60 \mathrm{kHz}$ and the pulse energy was set to $6.6 \pm 0.2 \mathrm{~mJ} /$ pulse. An effective incident value was obtained by considering the reflection and absorption losses in the mirrors and lenses. In all experiments of this study, the laser power sent to the test section is varied only by the pulse repetition frequency: the pulse energy was set to be constant. In these experiments, $Q$ can be evaluated in range of 10 to 100 .

\section{Drag Reduction Performance}

The drag reduction performance of repetitive laser pulse energy deposition up to $60 \mathrm{kHz}$ over a 10-mm-dia. blunt-cylinder-model under different Mach number conditions was studied experimentally using Mach-1.92-WT and Mach3.20-WT. A temporal history of the drag for Mach-3.20WT with a laser irradiation condition of $f_{\mathrm{e}}=50 \mathrm{kHz}$ is shown in Fig. 6. From this figure, drag was clearly decreased during laser irradiation. The baseline drag and standard deviation without energy deposition are tabulated in Table 2. The

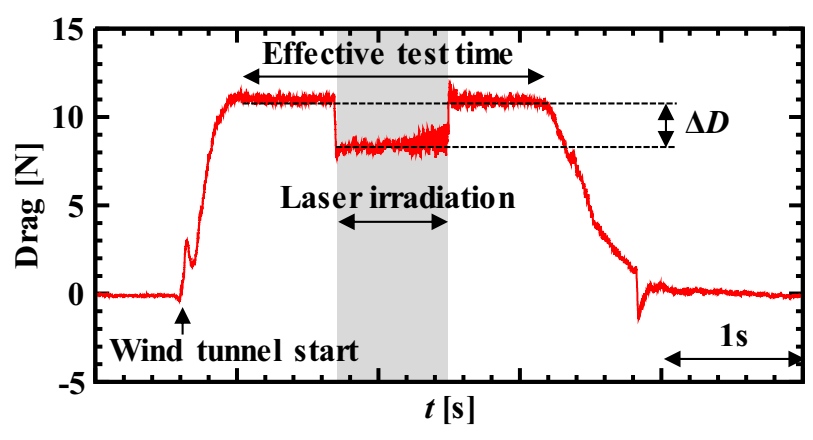

Fig. 6. Drag history of Mach-3.20 with $f_{\mathrm{e}}=50 \mathrm{kHz}$.

Table 2. Drag measured without energy deposition.

\begin{tabular}{ccc}
\hline & $D_{0}[\mathrm{~N}]$ & $\sigma[\mathrm{N}]$ \\
\hline Mach-1.92-WT & 10.4 & 0.02 \\
Mach-3.20-WT & 13.1 & 0.09 \\
\hline
\end{tabular}

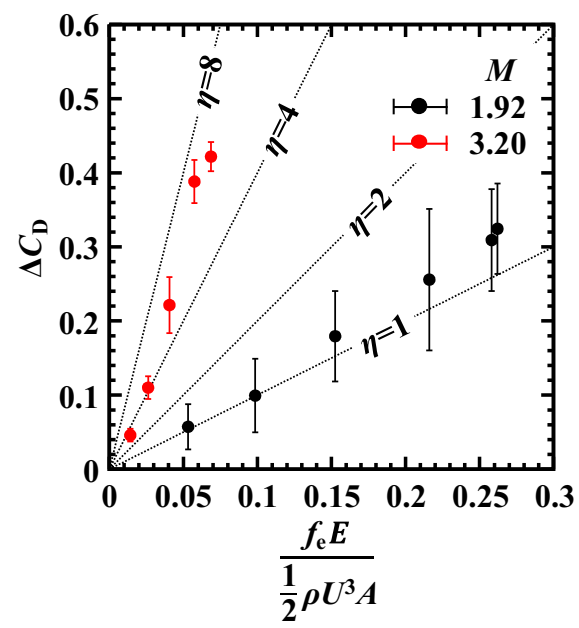

Fig. 7. Normalized drag reduction against normalized input power.

baseline drag for Mach-1.92 was $10.4 \mathrm{~N}$, and that for Mach-3.20 was $13.1 \mathrm{~N}$. The standard deviation in the drag at Mach-3.20 was larger than that at Mach-1.92. Next, drag with energy deposition was measured; the normalized drag reduction $\Delta C_{\mathrm{D}}$ was estimated using Eq. (3).

Figure 7 shows the normalized drag reduction against normalized power. The flow power input, that is the total enthalpy flux multiplied by the model projection, is incident on the model. In this figure, the efficiency of the energy deposition $\eta$ was evaluated using Eq. (4) and Eq. (5). The normalized drag reduction increases almost linearly with the normalized power; the increase rate at Mach-3.20 is larger than that at Mach-1.92.

\section{Discussion}

\subsection{Effects of Mach number}

In the following, the mechanism of improving drag reduction performance at higher Mach numbers will be discussed. Sequential Schlieren images with the respective Mach numbers during the interaction between a bow shock layer and a 


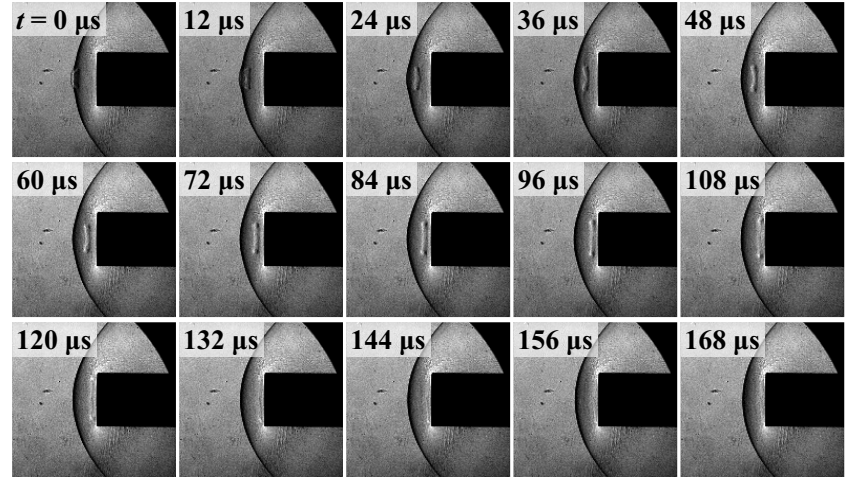

(a) $M=1.92$

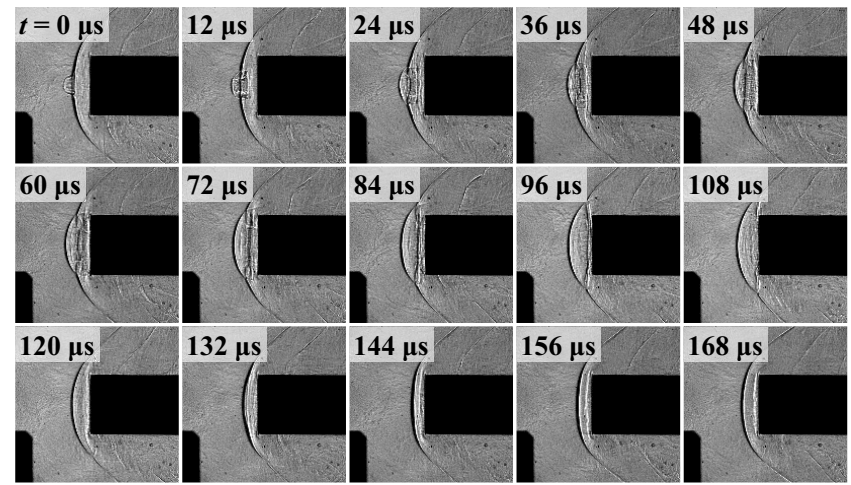

(b) $M=3.20$

Fig. 8. Snapshots of the Schlieren images.

single thermal bubble are shown in Fig. 8. With each Mach number, $t=0 \mu$ s corresponds to the instant when the thermal bubble attached the bow shock wave. In the Mach1.92 case, the vortex ring generated by the interaction between the thermal bubble and the shock-wave can be observed in the shock layer, and the behavior of this vortex ring in the shock layer can be clearly seen. However, in the Mach3.20 case, a vortex ring cannot be observed. The heating ratio $T_{\mathrm{h}} / T_{1}\left(=\rho_{1} / \rho_{\mathrm{h}}\right)$ per pulse can be estimated by these Schlieren images. Assuming the laser heating process is isochorical heating and the bubble expansion process is adiabatic expansion, the temperature of the thermal bubble is $469 \mathrm{~K}$ in the Mach-1.92 flow and $392 \mathrm{~K}$ in the Mach-3.20 flow. Using the static temperature of $163 \mathrm{~K}$ at Mach-1.92 and $107 \mathrm{~K}$ at Mach-3.20 as $T_{1}, T_{\mathrm{h}} / T_{1}$ per pulse is 2.9 in the Mach-1.92 flow and 3.9 in the Mach-3.20 flow.

Drag reduction performance can also described using an average heating ratio, ${ }^{19,29)} T_{\mathrm{h}} / T_{1}$, which is estimated for isobaric heating by

$$
\frac{T_{\mathrm{h}}}{T_{1}}=\frac{c_{\mathrm{p}} T_{1}+q_{\mathrm{b}}}{c_{\mathrm{p}} T_{1}}
$$

where $q_{\mathrm{b}}$ is the specific power input obtained by Eq. (28).

$$
q_{\mathrm{b}}=\frac{P_{\mathrm{b}}}{\rho_{1} U_{1} A_{\mathrm{b}}}
$$

$A_{\mathrm{b}}$ in Eq. (28) is the cross-sectional area of the thermal bubble. From the results of a reference test using a vacuum

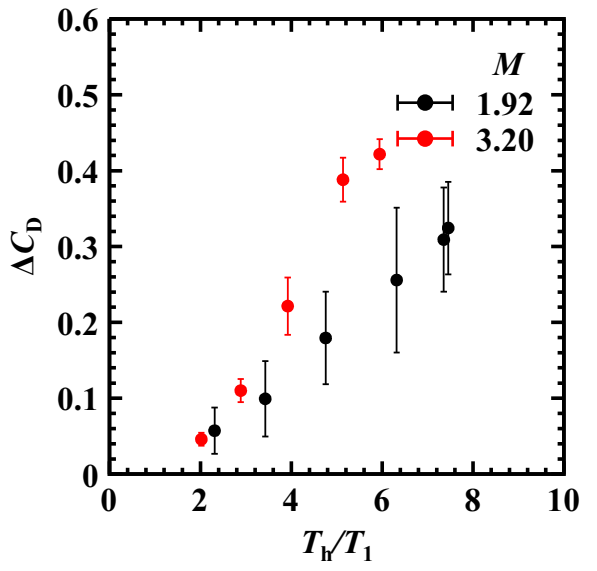

Fig. 9. Normalized drag reduction against heating ratio.

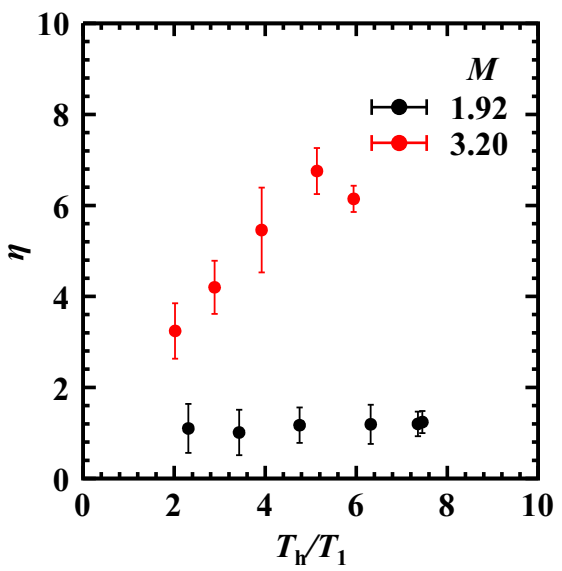

Fig. 10. Energy deposition efficiency against heating ratio.

chamber and the same laser optics, the initial size of the heating source under an ambient pressure of $30 \mathrm{kPa}$ can be estimated to be $1.0 \mathrm{~mm}$. Then, $T_{\mathrm{h}} / T_{1}$ can be obtained using this initial diameter. Figure 9 shows normalized drag reduction against the heating ratio. The normalized drag reduction tendency against the heating ratio was similar to the dependence on normalized input power, as shown in Fig. 7. Figure 10 shows the variation of $\eta$ against the average heating ratio. The $\eta$ of Mach-1.92 does not depend on the heating ratio, and was almost constant at 1.2. On the other hand, the $\eta$ of Mach-3.20 increased as the heating ratio rose. The maximum value of $\eta$ was achieved at 6.7. From these results, the drag reduction magnitude and efficiency of energy deposition improve as the Mach number increases. Hence, the drag reduction using repetitive pulse laser energy deposition works more effectively under the higher Mach number flow. These results are consistent with the results of Section 2.

The temporal histories of the shock stand-off distance at the centerline of the model evaluated from the Schlieren images are plotted in Fig. 11. The shock stand-off distance is normalized by a model diameter. The temporal history of the shock stand-off distance shows a similar tendency at both Mach numbers of 1.92 and 3.20. This is due to the so-called "lens effect" "26): the shock stand-off distance increases when 


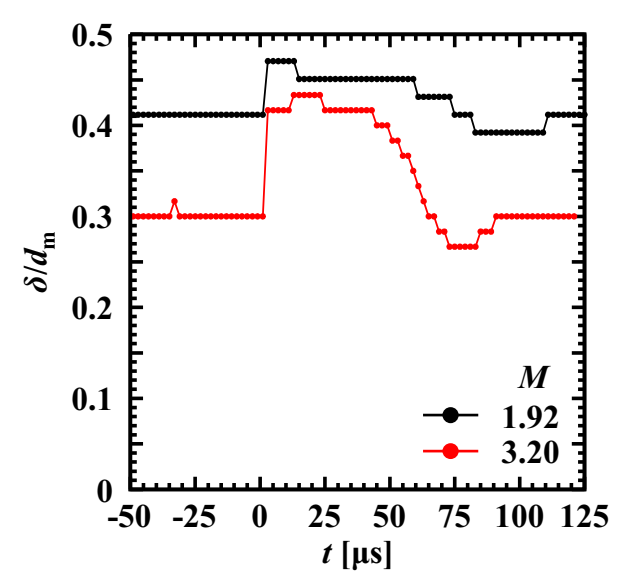

Fig. 11. Time history of the shock stand-off distance on center axis.

the interaction is initiated, and then decreases to a value smaller than the initial one. As seen by comparing Fig. 8 and Fig. 11, the shock stand-off distance stayed larger than the initial value in the period that the bubble or the vortex ring stayed in the bow shock layer. After the bubble or vortex ring left the shock layer, the shock stand-off distance became shorter than the initial value before it returned to the initial value.

The difference in Mach number appears in the duration time of the bow shock extrusion and the increase in shock stand-off distance induced by the lens effect. From Fig. 11, the duration of the bow shock extrusion in the Mach-1.92 flow is $80 \mu \mathrm{s}$ and the duration in the Mach-3.20 flow is $64 \mu \mathrm{s}$. The duration time should roughly correspond to the flow residence time, which is characterized by $d_{\mathrm{m}} / U$. The increase in maximum shock stand-off distance in the Mach1.92 flow is $15 \%$, and the maximum increment in the Mach-3.20 flow is $40 \%$. The increase in the maximum shock stand-off distance with the higher Mach number should be related to an increase in the counter-flow velocity, which is obtained as the solution of the Riemann problem in Section 2.

The shock stand-off distance on the centerline normalized by the model diameter against the repetition frequency normalized using the flow residence time, $d_{\mathrm{m}} / U$, is shown in Fig. 12. The circles plotted in Fig. 12 show the time-averaged shock stand-off distance, and the error bars in Fig. 12 show the maximum and minimum deflection in time variation. From Fig. 12, it can be seen that the averaged shock stand-off distances are larger as the repetition frequency increases for both Mach numbers. Under the experimental conditions, the shock stand-off distance increment in the Mach1.92 flow is about 30\% and that in the Mach-3.20 flow is about $60 \%$. The increment ratio of Mach-3.20 is approximately two times larger than that of Mach-1.92. The time variation of Mach-3.20 is larger than that of Mach-1.92. This corresponds to the shock-wave deformation characteristics induced by a single pulse, as shown in Fig. 8 .

\subsection{Effects of thermal bubble normalized diameter}

Because the normalized diameter of the thermal bubble $d_{\mathrm{h}} / d_{\mathrm{m}}$ is an important parameter for drag reduction using energy deposition, ${ }^{29)}$ drag reduction performance using two

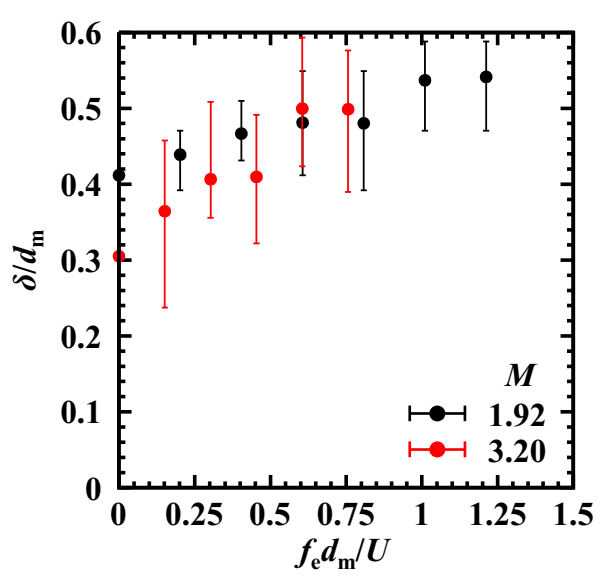

Fig. 12. Shock stand-off distance against repetition frequency.

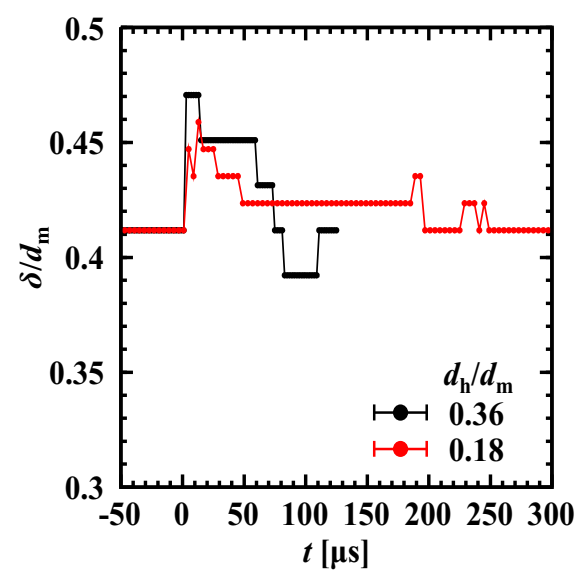

Fig. 13. Time history of the shock stand-off distance on center axis.

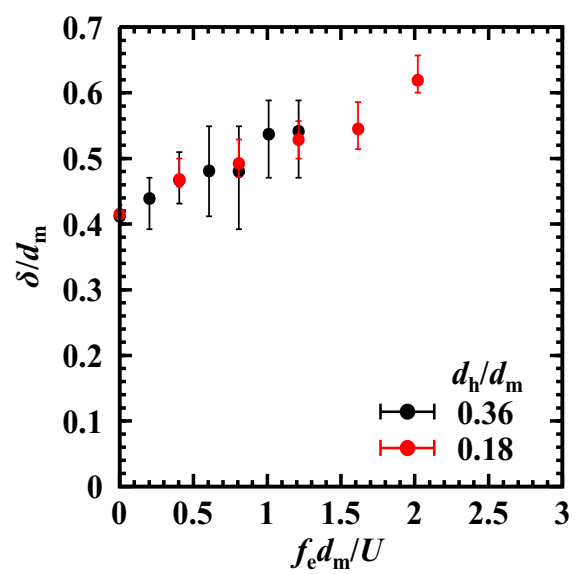

Fig. 14. Shock stand-off distance against repetition frequency.

model diameters of 10 and $20 \mathrm{~mm}$ under the same energy deposition condition were examined in the Mach-1.92 flow. The $d_{\mathrm{h}} / d_{\mathrm{m}}$ was 0.36 and 0.18 , respectively, because the incident bubble diameter $d_{\mathrm{h}}$ estimated using Schlieren images is $3.6 \mathrm{~mm}$ in the Mach-1.92 flow. The temporal history of the shock stand-off distance with single-pulse energy deposition and the time-averaged shock stand-off distance against the repetition frequency are shown in Fig. 13 and Fig. 14, re- 


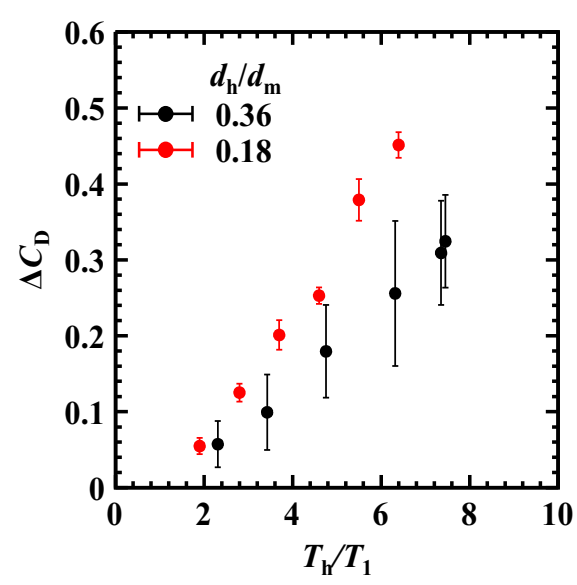

Fig. 15. Normalized drag reduction against heating ratio.

spectively. In Fig. 13, the history of the normalized shock stand-off distance variation strongly depends on the model diameter. In the case of $d_{\mathrm{m}}=20 \mathrm{~mm}\left(d_{\mathrm{h}} / d_{\mathrm{m}}=0.18\right)$, the peak value of the normalized shock stand-off distance is smaller but the duration of the extrusion is longer. On the other hand, from Fig. 14, the normalized shock stand-off distance based on the model diameter and flow speed does not depend on the model diameter. These results can be interpreted as follows. If the Mach number is the same, the normalized shock stand-off distance $\delta / d_{\mathrm{m}}$ is constant because the flow field is similar. The remaining time of the vortex ring in the shock layer $\tau$ is determined by $U / \delta$, where $U$ is the flow speed. Therefore, $\tau$ is proportional to $d_{\mathrm{m}}$. From Figs. 8 and 13, the shock-wave is deformed in the period that the vortex ring stays in the bow shock layer. Hence, the duration of shock-wave extrusion is longer with the larger model diameter. On the other hand, the shockwave deformation magnitude does not depend on the model diameter because the magnitude is determined mainly by the condition in the thermal bubble and the strength of the shock-wave.

The drag reduction performance and efficiency of energy deposition against the heating ratio are shown in Fig. 15 and Fig. 16, respectively. Both the drag reduction and efficiency of energy deposition are improved with smaller value for $d_{\mathrm{h}} / d_{\mathrm{m}}$ or when the model diameter is increased; in particular, $\eta$ is drastically improved. This dependence of drag reduction on the normalized diameter of the thermal bubble is consistent with a previous study. ${ }^{32)}$ These results indicate that the model dimension affects the vortex ring remaining time, and drag reduction performance strongly depends on the bubble residence time in the shock layer. Hence, further improvement in drag reduction performance using repetitive energy deposition can be achieved using a larger model. Or, for a given model dimension, a small amount of energy can be efficiently input for drag reduction. This implies that over the whole part of a flying body, this energy deposition scheme is expected to be efficiently done not by depositing a large amount of power to a specific location but by widely distributing a small amount of deposited powers to many locations.

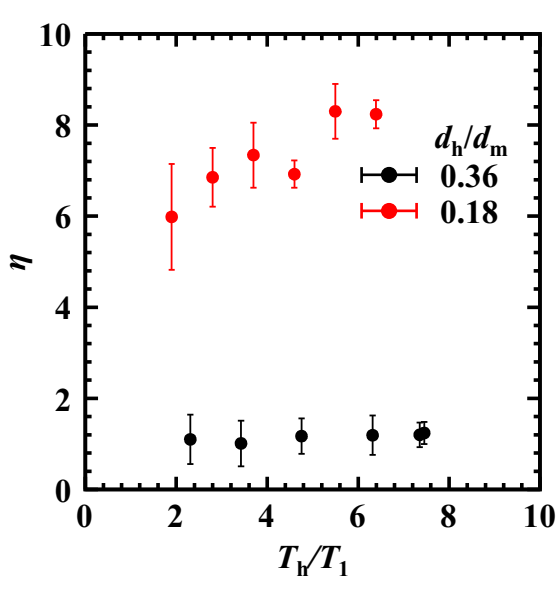

Fig. 16. Energy deposition efficiency against heating ratio.

In the present study, because of facility size limitations the model diameter effect could not be investigated in the Mach3.20-WT, in which a much more efficient drag reduction performance is expected.

\section{Conclusion}

Drag reduction performance using repetitive laser energy deposition over the same blunt body at two Mach numbers was experimentally investigated. The drag reduction and efficiency of energy deposition at each Mach number were increased as repetition frequency increased. The normalized drag reduction $\Delta C_{\mathrm{D}}$ increased from $6 \%$ in the Mach-1.92 flow to $40 \%$ in the Mach-3.20 flow with the same heating ratio; the maximum efficiency of energy deposition was improved from 1.2 to 6.7. Therefore, better drag reduction performance using repetitive energy deposition was obtained with the higher Mach number. From the visualization results of single-pulse energy deposition, the shock-wave extrusion due to the lens effect was increased from 15\% at Mach-1.92 to $40 \%$ at Mach-3.20, although the duration of the shockwave extrusion was shortened from $80 \mu \mathrm{s}$ at Mach-1.92 to $64 \mu$ s at Mach-3.20.

In addition, under the same Mach number and energy deposition scheme, the drag reduction and efficiency of energy deposition improved as the normalized diameter of the thermal bubble decreased; the normalized drag reduction increased from $6 \%$ with the 10 -mm-dia. model $\left(d_{\mathrm{h}} / d_{\mathrm{m}}=0.36\right)$ to $45 \%$ with the $20-\mathrm{mm}$-dia. model $\left(d_{\mathrm{h}} / d_{\mathrm{m}}=0.18\right)$, and the maximum efficiency of energy deposition improved from 1.2 with the 10 -mm-dia. model to 8.2 with the 20 -mm-dia. model. These improvements in normalized diameter were consistent with extending the vortex ring residence time in the shock layer.

From these results, it has been experimentally demonstrated that drag reduction performance using repetitive laser energy deposition is improved at higher Mach numbers. Additionally, it has been confirmed that decreasing the normalized diameter also improves drag reduction performance under same Mach number and same energy deposition scheme. 


\section{Acknowledgments}

The authors would like to grateful thank Prof. I. Funaki, Institute of Space and Astronautical Science, Japan Aerospace Exploration Agency, Dr. S. Watanabe, Dr. K. Mitsuo, Dr. K. Fujii and Dr. S. Nagai, Aeronautical Technology Directorate, Japan Aerospace Exploration Agency, for their assistance to design the wind tunnel. The authors would also like to gratefully thank Messrs. A. Saito, M. Nakakimura and K. Tachibana, Technical Division, Nagoya University, for their assistance to manufacture the wind tunnel. This research was supported by the Japan Society for Promotion of Science, Grant-in-Aid for Scientific Research (A), No. 15H02321.

\section{References}

1) Bushnell, D. M.: Shock Wave Drag Reduction, Annu. Rev. Fluid Mech., 36 (2004), pp. 81-96.

2) Knight, D. D.: Survey of Aerodynamic Drag Reduction at High Speed by Energy Deposition, J. Propul. Power, 24 (2008), pp. 1153-1167.

3) Georgievskii, P. and Levin, V.: Supersound Body Bypass with External Resources of Heat Release, Pisma v Zhurnal Tekhnicheskoi Fiz., 14 (1988), pp. 684-687.

4) Tret'yakov, P. K. and Garanin, A. F.: Control of Supersonic Flow around Bodies by Means of High-Power Recurrent Optical Breakdown, Physics-Doklay, 41 (1996), pp. 566-597.

5) Erdem, E., Kontis, K., and Yang, L.: Steady Energy Deposition at Mach 5 for Drag Reduction, Shock Waves, 22 (2012), pp. 285-298.

6) Sasoh, A., Sekiya, Y., Sakai, T., Kim, J.-H., and Matsuda, A.: Supersonic Drag Reduction with Repetitive Laser Pulses through a Blunt Body, AIAA J., 48 (2010), pp. 2811-2817.

7) Kim, J.-H., Matsuda, A., Sakai, T., and Sasoh, A.: Wave Drag Reduction Performance with Acting Spike Induced by Laser-Pulse Energy Depositions, AIAA J., 49 (2011), pp. 2076-2078.

8) Satheesh, K. and Jagadeesh, G.: Experimental Investigations on the Effect of Energy Deposition in Hypersonic Blunt Body Flow Field, Shock Waves, 18 (2008), pp. 53-70.

9) Satheesh, K. and Jagadeesh, G.: Effect of Concentrated Energy Deposition on the Aerodynamic Drag of a Blunt Body in Hypersonic Flow, Phys. Fluids, 19 (2007), 31701.

10) Schulein, E. and Zheltovodov, A.: Effects of Steady Flow Heating by Arc Discharge Upstream of Non-Slender Bodies, Shock Waves, 21 (2011), pp. 383-396.

11) Zudov, V. N., Tret'yakov, P. K., Tupikin, A. V., and Yakovlev, V. I.: Supersonic Flow Past a Thermal Source, Fluid Dyn., 38 (2003), pp. $782-793$.

12) Adelgren, R. G., Yan, H., Elliott, G. S., Knight, D. D., Beutner, T. J., and Zheltovodov, A. A.: Control of Edney IV Interaction by Pulsed Laser Energy Deposition, AIAA J., 43 (2005), pp. 256-269.

13) Sasoh, A., Kim, J.-H., Yamashita, K., and Sakai, T.: Supersonic Aerodynamic Performance of Truncated Cones with Repetitive Laser Pulse Energy Depositions, Shock Waves, 24 (2013), pp. 59-67.

14) Borzov, V. Y., Rybka, I. V., and Yur'ev, A.: Effect of Local Energy Supply to a Hypersonic Flow on the Drag of Bodies with Different Nose Bluntness, J. Eng. Phys. Thermophys., 67 (1995), pp. 997-1002.

15) Ogino, Y., Ohnishi, N., Taguchi, S., and Sawada, K.: Baroclinic Vor- tex Influence on Wave Drag Reduction Induced by Pulse Energy Deposition, Phys. Fluids, 21 (2009), 66102.

16) Sakai, T.: Supersonic Drag Performance of Truncated Cones with Repetitive Energy Depositions, Int. J. Aerosp. Innov., 1 (2009), pp. 31-44.

17) Riggins, D., Nelson, H. F., and Johnson, E.: Blunt-Body Wave Drag Reduction using Focused Energy Deposition, AIAA J., 37 (1999), pp. 460-467.

18) Riggins, D. W. and Nelson, H. F.: Hypersonic Flow Control using Upstream Focused Energy Deposition, AIAA J., 38 (2000), pp. 4-6.

19) Georgievskii, P. Y. and Levin, V. A.: Control of the Flow Past Bodies Using Localized Energy Addition to the Supersonic Oncoming Flow, Fluid Dyn., 38 (2003), pp. 794-805.

20) Anderson, K. and Knight, D. D.: Interaction of Heated Filaments with a Blunt Cylinder in Supersonic Flow, Shock Waves, 21 (2011), pp. 149-161.

21) Kremeyer, K., Ebastian, K., and Shu, C.-W.: Computational Study of Shock Mitigation and Drag Reduction by Pulsed Energy Lines, AIAA J., 44 (2006), pp. 1720-1731.

22) Zaidi, S. H., Shneider, M. N., and Miles, R. B.: Shock-Wave Mitigation through Off-Body Pulsed Energy Deposition, AIAA J., 42 (2004), pp. 326-331.

23) Shneider, M. N., Macheret, S. O., Zaidi, S. H., Girgis, I., and Miles, R. B.: Virtual Shapes in Supersonic Flow Control with Energy Addition, J. Propul. Power, 24 (2008), pp. 900-915.

24) Furukawa, D., Aoki, Y., Iwakawa, A., and Sasoh, A.: Moderation of Near-Field Pressure over a Supersonic Flight Model Using Laser-Pulse Energy Deposition, Phys. Fluids, 28 (2016), 51701.

25) Iwakawa, A., Sakai, T., and Sasoh, A.: Repetition Frequency Dependence of Wave Drag Reduction Induced by Laser-Pulse-Energy Depositions, Trans. JSASS Aerospace Technology Japan, 11 (2013), pp. 53-60.

26) Georgievskii, P. Y. and Levin, V. A.: Unsteady Interaction of a Sphere with Atmospheric Temperature Inhomogeneity at Supersonic Speed, Fluid Dyn., 28 (1993), pp. 568-574.

27) Richtmyer, R. D.: Taylor Instability in Shock Acceleration of Compressible Fluids, Commun. Pure Appl. Math., XIII (1960), pp. 297-319.

28) Tret'yakov, P., Garanin, A., Kraynev, V., Tupikin, A., and Yakovlev, V.: Investigation of Local Laser Energy Release Influence on Supersonic Flow by Methods of Aerophysical Experiments, Int. Conf. Methods Aerophysical Res., 1996, pp. 200-203.

29) Schulein, E.: Simplified Model for Flow-Heating Effect on Wave Drag and Its Validation, AIAA J., 54 (2016), pp. 1030-1039.

30) Toro, E. F.: Riemann Solvers and Numerical Method for Fluid Dynamics, Springer Verlag, Berlin, 1999.

31) Iwakawa, A., Osuka, T., Shoda, T., Sasoh, A., and Kawazoe, H.: RingForce Balance System for Small Wind Tunnels, Trans. JSASS Aerospace Technology Japan, 13 (2015), pp. 51-60.

32) Lashkov, V. A., Mashek, I. Ch., Anisimov, Y. I., Ivanov, V. I., Kolesnichenko, Y. F., Ryvkin, M. I., and Gerynya, A. A.: Gas Dynamic Effect of Microwave Discharge on Supersonic Cone-Shaped Bodies, 42nd AIAA Aerosp. Sci. Meet. Exhib., 2004, AIAA 2004-671.

N. Ohnishi

Associate Editor 